\title{
Hierarchical Self-Assembly of Cellulose Nanocrystals in a Confined Geometry
}

\author{
Richard M. Parker, ${ }^{\dagger}$ Bruno Frka-Petesic, $^{\dagger}$ Giulia Guidetti, Gen Kamita, Gioele Consani, Chris Abell, \\ and Silvia Vignolini*
}

Department of Chemistry, University of Cambridge, Lensfield Road, Cambridge CB2 1EW, United Kingdom

\author{
Supporting Information
}

\begin{abstract}
Complex hierarchical architectures are ubiquitous in nature. By designing and controlling the interaction between elementary building blocks, nature is able to optimize a large variety of materials with multiple functionalities. Such control is, however, extremely challenging in man-made materials, due to the difficulties in controlling their interaction at different length scales simultaneously. Here, hierarchical cholesteric architectures are obtained by the self-assembly of cellulose nanocrystals within shrinking, micron-sized aqueous droplets. This confined, spherical geometry drastically affects the colloidal self-assembly process, resulting in concentric ordering within the droplet, as confirmed by simulation. This provides a quantitative tool to study the interactions of cellulose nanocrystals beyond what has been achieved in a planar geometry. Our developed methodology allows us to fabricate truly hierarchical solid-state architectures from the nanometer to the macroscopic scale using a renewable and sustainable biopolymer.
\end{abstract}

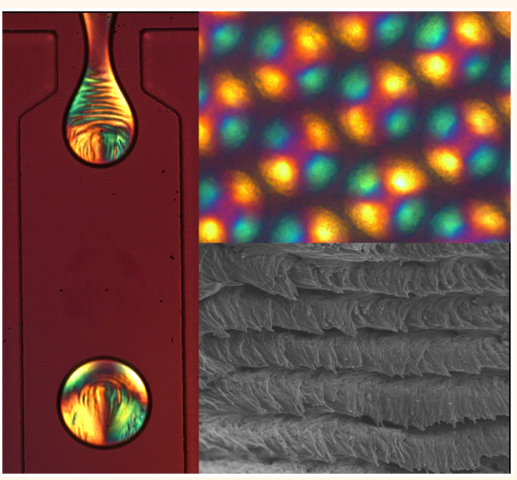

KEYWORDS: liquid crystals, microfluidics, colloidal self-assembly, cellulose nanocrystals, hierarchical architecture

$\mathrm{D}$ esigning and controlling the interaction of elementary building blocks into complex hierarchical architectures enables nature to optimize a large variety of materials with multiple functionalities. ${ }^{1-3}$ Within the fascinating variety of designs and materials, helicoidal architectures are a recurring motif in nature, ${ }^{4-8}$ yet little is known about the natural assembly process. ${ }^{9}$ Consequently, the ability to fabricate biomimetic replicas is fundamentally important not only to elucidate the design principles that underlie the evolution of complex hierarchical structures in nature but also to inspire the development of materials where their functionalities are encoded in the building blocks that compose them. ${ }^{10-12}$ However, control across a diversity of length scales ranging from nanometers to several hundreds of microns is extremely challenging in man-made materials. ${ }^{13}$

Here, inspired by the helicoidal architectures found in the plant cell wall, ${ }^{14}$ we study the assembly of cholesteric architectures of cellulose nanocrystals (CNCs) confined within monodisperse micron-sized droplets, generated via dropletbased microfluidics. ${ }^{15,16}$ The self-assembly process is monitored with polarized optical microscopy, and the effect of the initial aqueous $\mathrm{CNC}$ concentration on the resultant self-assembled architecture is studied. This system not only allows for the localized, quantitative investigation of the complex dynamic interaction of CNCs in suspension but also offers a pathway to obtain highly hierarchical structures in a confined geometry from the nanometer to the macroscopic scale, using a renewable and sustainable polymer.
Exploitation of cellulose has existed since the beginning of civilization, from clothes and paper to use as a construction material, ${ }^{17}$ yet over the last two decades, it has attracted a growing interest due to its abundance and versatility when processed on the nanoscale in the form of cellulose nanocrystals. ${ }^{18}$ By strong acid hydrolysis, CNC can be extracted from a variety of natural sources, producing stable aqueous suspensions that exhibit cholesteric liquid-crystalline behavior at higher concentrations. ${ }^{19}$ The evaporation of CNC suspensions on a flat substrate can result in the formation of a solid film with a periodic chiral structure that can reflect visible light. ${ }^{20}$ Traditionally, the self-assembly of such colloidal liquid-crystal systems has been studied in planar geometries, ${ }^{21-23}$ offering a large variety of applications, for example, in pressure or temperature sensors. ${ }^{24,25}$ However, more recently, there has been increasing study into the effects of topological constraints. ${ }^{26}$ Of particular interest is the spherical geometry, where the curvature imposed, for example, by the interface of an emulsified droplet leads to frustrated liquid-crystalline self-organization. ${ }^{27}$ This spherical topology, either as a droplet or as a thin shell of liquid, has been shown to give rise to peculiar phenomena, with potential applications in actuators or lasers. ${ }^{16,28,29}$

Received: May 20, 2016

Accepted: August 26, 2016

Published: August 26, 2016 

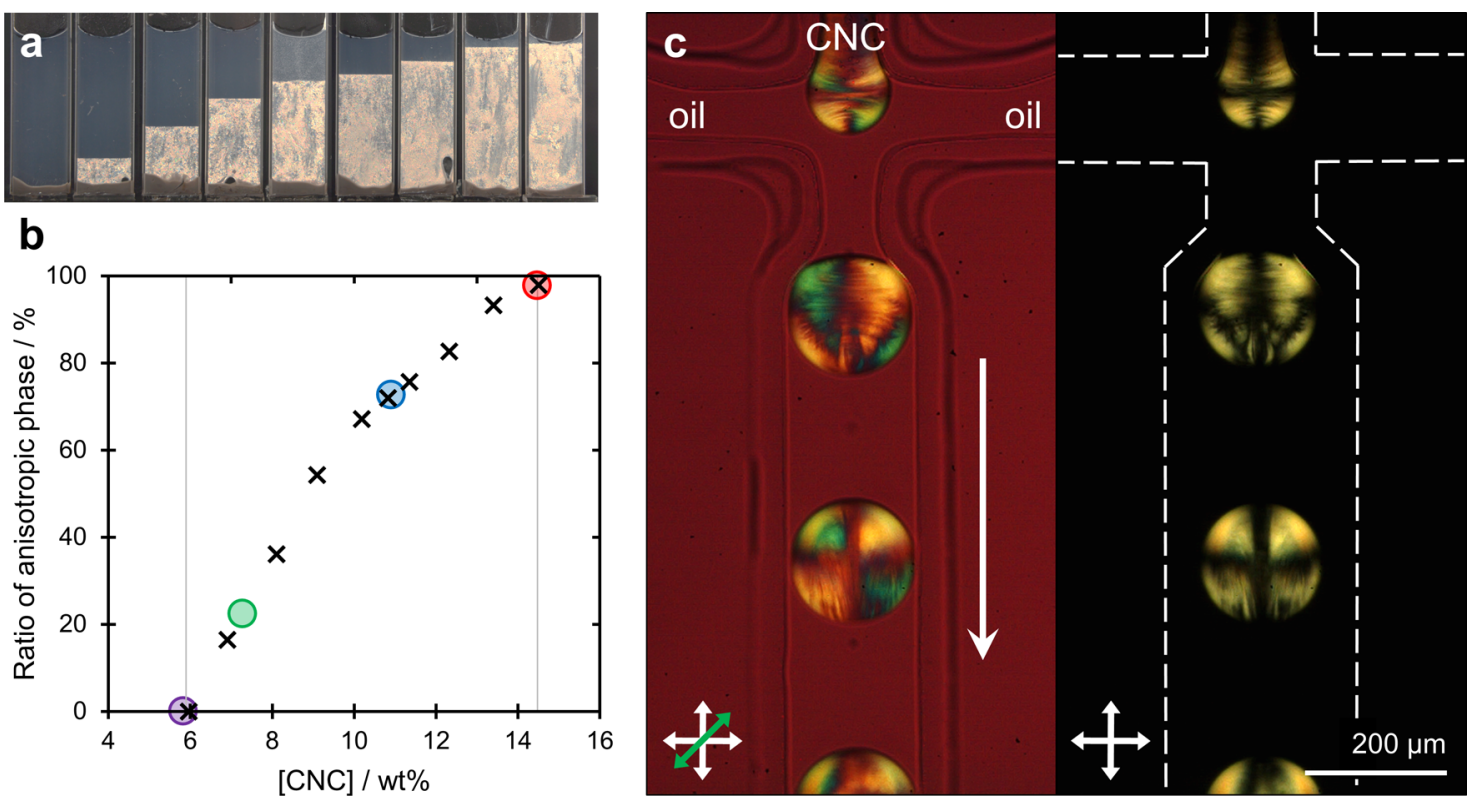

Figure 1. (a) Phase behavior of CNC suspensions of increasing concentration, as imaged under cross-polarizers, where a clear transition from pure isotropic to anisotropic phase is observed from left to right. (b) Calculated ratio of anisotropic phase present for each concentration investigated in (a) was used to compile the phase diagram (crosses). The specific concentrations investigated within microdroplets are indicated by the colored circles. (c) Polarization micrograph of the generation of microfluidic water-in-oil droplets from a 14.5 wt $\%$ suspension of CNCs, as imaged under cross-polarizers (right) and with a first-order tint plate (left), illustrating the initial radial assembly.

The study of the self-assembly of CNCs in a microdroplet enables (i) the reproducible measurement of the cholesteric pitch at concentrations that are inaccessible with traditional pitch diagrams due to kinetic arrest, and (ii) monitoring of the self-assembly process over a few hours, instead of several days or weeks, ${ }^{30}$ thereby reducing errors induced by desulfation of the $\mathrm{CNC} .^{31}$

\section{RESULTS AND DISCUSSION}

An aqueous suspension of cellulose nanocrystals (Figure S1, Supporting Information) was prepared as described in the Experimental Methods. To characterize the lyotropic properties of this suspension, it was diluted to give a series of CNC concentrations from 14.5 to $4.7 \mathrm{wt} \%$, and the proportion of anisotropic phase was evaluated at each concentration (Figure 1a). This enabled the construction of a traditional phase diagram, as shown in Figure 1b. This phase diagram allows for determination of the critical values of $\mathrm{CNC}$ concentration for this specific suspension at which the transition from the isotropic to the anisotropic phase occurs.

In order to understand the impact of geometrical confinement, it was necessary to study how the initial concentration of the CNC suspension affected self-assembly within a micronscale droplet. Microdroplets were generated in a single step as an aqueous emulsion in hexadecane oil within a polydimethylsiloxane (PDMS) flow-focusing microfluidic device, as described in the Experimental Methods. At the flow-focusing junction, the aqueous CNC suspension intersected perpendicularly with flows of hexadecane oil, resulting in segmentation into monodisperse microdroplets (coefficient of variation $<2 \%$ ), with a diameter defined by the geometry of the flow focus, and the relative flow rates and viscosities of the immiscible solutions. Once formed, the droplets were collected via microbore tubing onto a fluorophilic substrate for further study. It should be noted that the aqueous droplets are denser than the surrounding oil and, as such, settle onto the surface of the fluorophilic substrate rather than at the air-oil interface (Figure S2, Supporting Information). The presence of this oil layer slows the loss of water from the droplets, allowing them to be studied over time scales from hours to days.

Microdroplets with a typical diameter of $140 \mu \mathrm{m}$ were prepared from a series of $\mathrm{CNC}$ concentrations across the phase transition, as indicated by the colored circles in Figure $1 \mathrm{~b}$. In all cases, the optical anisotropy of the suspension allowed for the ordering of $\mathrm{CNC}$ domains to be visualized during droplet formation by polarized optical microscopy, as described in the Experimental Methods. This is exemplified with a 14.5 wt \% CNC suspension, as denoted by the red circle in the phase diagram (Figure 1b). Here, the generation of an interface between the two fluids at the neck of the flow focus resulted in an immediate radial ordering of the CNCs within the microdroplet, giving rise to a pronounced Maltese cross-like pattern when imaged under crossed polarizers (Figure 1c; see Video S1 and Video S2). However, this arrangement is rapidly disrupted by chaotic advection induced within the microdroplet by shearing interactions with its surroundings as it travels along the microfluidic channel. At such a high concentration, the suspension is highly viscous and is almost entirely in a liquidcrystalline phase. For this reason, the shear experienced by the cholesteric phase inside the droplet induces many topological defects. This droplet consequentially remains trapped in this disordered anisotropic state without any particular reordering of the structure. Upon subsequent removal of water (by diffusion into the oil) and corresponding concentration of the confined suspension, this arrangement is preserved (Figure S3, Supporting Information). A similar trend is observed for a lower concentration of 10.9 wt \% (blue circle on Figure $1 \mathrm{~b}$ ). Here, despite microdroplets containing both isotropic and anisotropic phases, the relaxation toward a radial geometry was again not significantly observed after initial droplet formation (Figure S4, Supporting Information). 

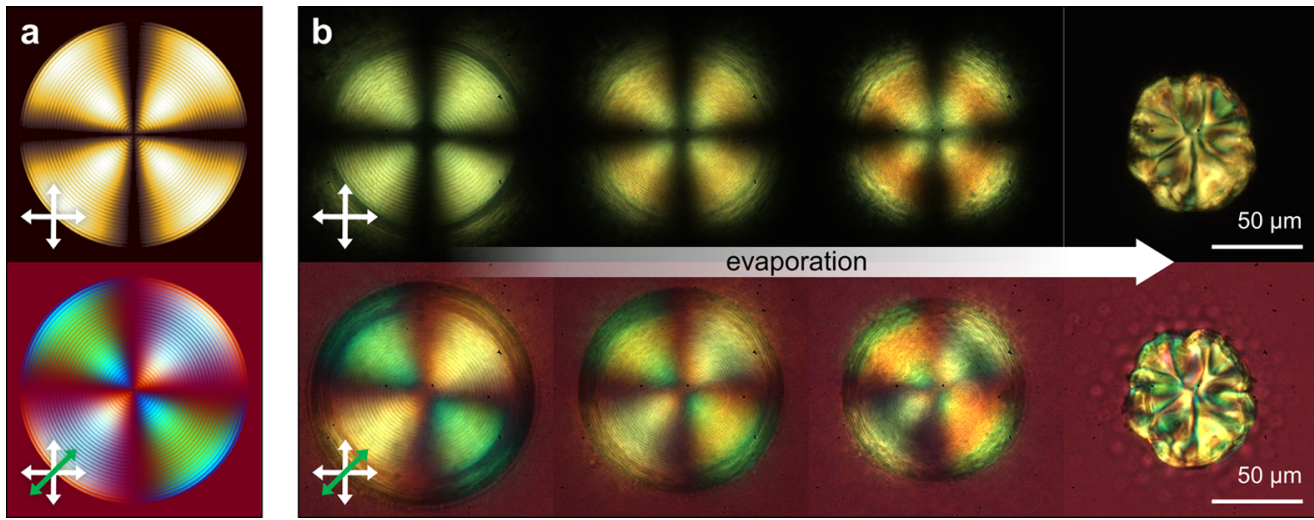

Figure 2. Comparison between (a) theoretical and (b) experimental images obtained from the confinement of a cholesteric CNC suspension within a spherical geometry, when viewed through cross-polarizers (top row) and upon addition of a first-order tint plate (bottom row). Upon loss of water, the Maltese cross is retained until the onset of buckling upon final drying.

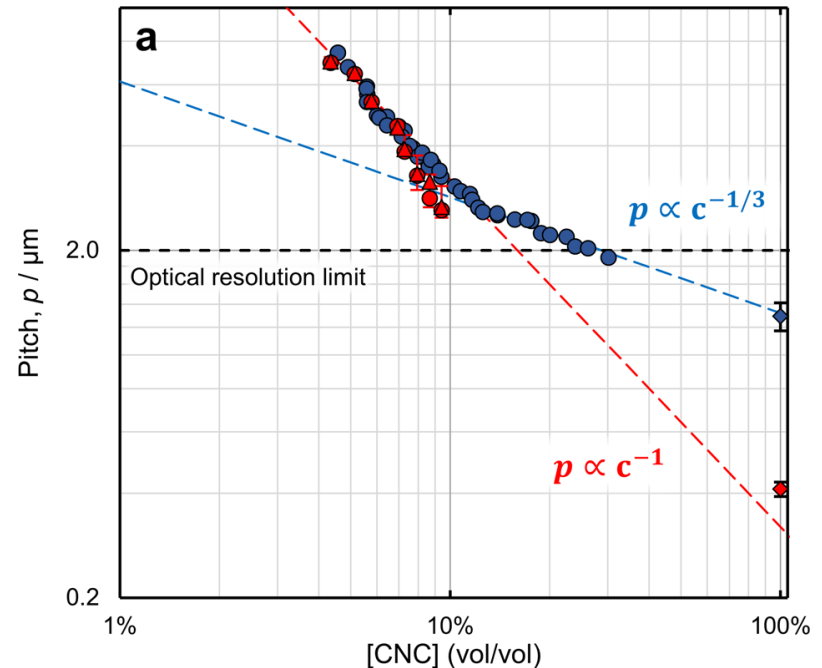

b
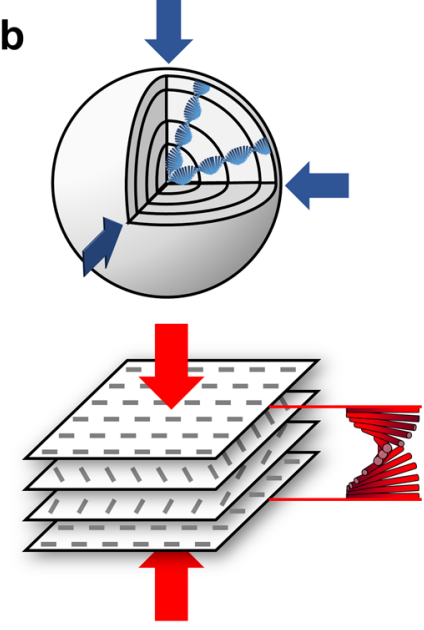

Figure 3. (a) Evolution pitch diagram. The cholesteric pitch measured in the droplets (blue circles) is compared against a macroscale capillary measured by laser diffraction (red circles) and microscopy (red triangles). The pitch below $2 \mu \mathrm{m}$ was not measured due to the optical resolution limit. Upon increasing CNC concentration, the measured cholesteric pitch in the droplets is initially consistent with the pitch measured in the capillary; however, for the case of confined suspensions, a transition at $c_{\mathrm{g}}=12 \% \mathrm{v} / \mathrm{v}(\sim 19 \mathrm{wt} \%)$ is observed. Extrapolation of the $c^{-1}$ and $c^{-1 / 3}$ trend lines correlates with the pitch measured by scanning electron microscopy for a dry film and microparticle, respectively (diamonds). The capillary error bars correspond to the gradient in pitch observed as a function of position within the anisotropic phase, as shown in Figure S16, Supporting Information. (b) Schematics illustrating the effect on the helicoidal cholesteric structure upon threedimensional contraction when confined within a sphere, as occurs after $c_{\mathrm{g}}\left(p \propto c^{-1 / 3}\right.$, top), compared to unidirectional contraction in a planar geometry $\left(p \propto c^{-1}\right.$, bottom).

A markedly different assembly process is observed when the isotropic phase is dominant within the microdroplet, as is the case for $7.3 \mathrm{wt} \%$ suspension of CNCs (green circle in Figure 1b). As before, radial ordering of the liquid-crystalline structure is observed upon generation of the microfluidic droplets; however, mixing within the droplet as it flows along the channel results in microdroplets in a predominantly isotropic phase, containing clearly defined tactoids (Figure S5, Supporting Information). Upon the loss of water from the droplet the tactoids rearrange, resulting in the formation of an ordered chiral nematic shell, growing inward from the water-oil interface (Figure 2). Depending on the number and dimensions of the tactoids (which is influenced by the individual composition of each microdroplet), either a chiral nematic shell containing free tactoids or a radial order throughout the entire diameter of the droplet is obtained (Figure S6, Supporting Information). In the latter case, such ordering can be preserved until the onset of buckling during the final stages of water loss from the droplet (Figure S7, Supporting Information). We attribute buckling to the interplay between increasing Laplace pressure at lower radius of curvature and the resistance to isotropic compression of the solidified CNC shell of the microdroplet, which allows for loss of the remaining water content without laterally compressing the rigidified surface. The presence of the chiral nematic organization throughout the entire diameter of the droplet is confirmed by the numerically simulated pattern shown in Figure 2a (see Supporting Information for simulation details). In particular, the observation of concentric dark and bright circles corresponds to the fingerprint pattern of the cholesteric structure. The superimposed Maltese cross pattern is due to the isoclines of the radial cholesteric helix axis aligned with the axes of the crossed polarizers, in agreement with a planar anchoring of the $\mathrm{CNC}$ local director with the droplet interface. 
Low viscosity and a homogeneous composition are expected to increase the proportion of droplets retaining the radial chiral nematic order, with the in situ formation of a chiral nematic phase expected to reduce the generation of shear-induced topological defects. This was confirmed with droplets solely containing an isotropic suspension of CNC (5.8 wt \%, purple circle in Figure 1b), where the formation of independent tactoids within the volume of the droplet was no longer observed upon droplet shrinkage, with the isotropicanisotropic phase transition instead initiated exclusively at the water-oil interface (Figure S8, Supporting Information). Furthermore, lower concentrations have been tested. However, for values lower than $4 \mathrm{wt} \%$, the loss of water in ambient conditions leads only to a thin shell with transient cholesteric order. This shell is subsequently disrupted by buckling ${ }^{32}$ prior to the droplet core becoming sufficiently concentrated.

The observation of the microdroplet shrinkage process by polarized optical microscopy allowed for monitoring of the pitch evolution as a function of the water content. This particular configuration enables the construction of an "evolution pitch diagram" which depicts the behavior of the cholesteric phase from low concentration at the equilibrium toward the final dry state. The pitch $(p)$ is measured as twice the periodicity of the fingerprint pattern observed between crossed polarizers, while the suspension concentration is calculated from the droplet diameter. Variation in the pitch measured across a single droplet was within experimental error, indicating that the $\mathrm{CNC}$ concentration was uniform throughout the shrinking droplet. Similarly, droplets with comparable CNC concentration displayed the same cholesteric pitch. The values of the pitch measured during the loss of water from the microdroplets are reported in Figure 3 and compared to the values of the pitch independently obtained from measurement in a glass capillary (see Experimental Methods). It is worth noting here that, for a given initial value of $\mathrm{CNC}$ concentration, the same trend in pitch is measured irrespective of the dimensions of the initial droplets (Figure S9, Supporting Information).

The essence of the self-assembly process of $\mathrm{CNC}$ in suspension is illustrated by Figure 3. At a low concentration of CNC in suspension, the cholesteric pitch observed both in the droplets and in the capillaries overlaps and appears to be inversely proportional to concentration, $p \propto c^{-1}$, as expected from Straley modeling of chiral nonflexible rods. ${ }^{33}$ The small discrepancy between the two pitch measurements above $9.7 \mathrm{wt}$ $\%$ is attributed to the cholesteric monodomain within the droplets, as commented upon in the Supporting Information. In contrast, droplets prepared from $[\mathrm{CNC}]=10.9 \mathrm{wt} \%$ remain trapped in a polydomain structure and, as such, more closely follow the capillary data (Figure S10).

Significantly, above a critical concentration denoted here as $c_{\mathrm{g}}$, a transition after which the pitch scales as $p \propto c^{-1 / 3}$ is observed. This transition is attributed to the manifestation of the kinetic arrest, where the sample cannot relax. Indeed, such kinetic arrest is expected to take place at some point during shrinkage toward a fully dried sphere of self-assembled CNCs. The value of $c_{\mathrm{g}}$ for this specific suspension $(12 \% \mathrm{v} / \mathrm{v} \approx 19 \mathrm{wt} \%$ in Figure 3) was found to be comparable between individual radially ordered cholesteric droplets, irrespective of the initial size, CNC concentration, or rate of water removal. The concentration at which this transition occurs has been discussed and addressed in the literature as a key factor in the understanding of the self-assembly of CNCs but remains challenging to assess. ${ }^{34}$ As the suspension gets kinetically trapped, the cholesteric structure cannot relax over time, but it still can be affected by the local shear experienced upon drying, and therefore, it is sensitive to any geometrical constraints. In this system, the spherical geometry leads to a three-dimensional contraction of the cholesteric structure, in agreement with the observed power law. This contrasts with the usual configuration where a film is cast onto a planar substrate, which leads to a unidirectional, vertical straining of the cholesteric structure and consequentially to a similar power law of $p \propto c^{-1}$. For this reason, the spherical geometry allows for a clear discrimination of the transition upon kinetic arrest.

Finally, Figure 4 shows the morphology of CNC microparticles after the complete loss of water. For this experiment,

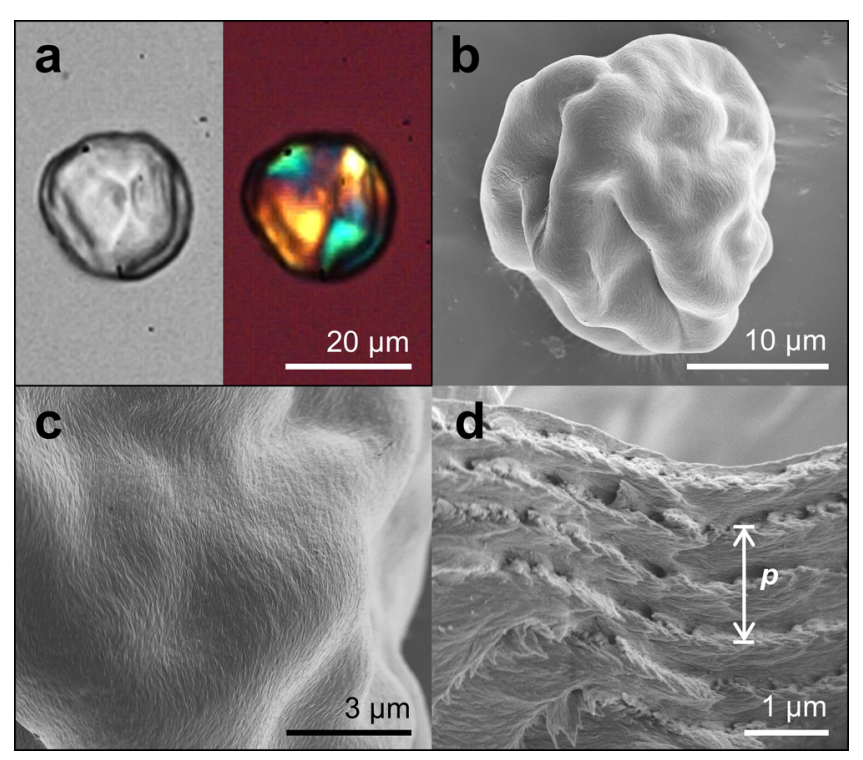

Figure 4. (a) Image of a dried CNC microparticle, as imaged in transmission (left) and under cross-polarizers with a first-order tint plate (right). (b-d) SEM images of a dry, buckled CNC microparticle, showing (c) the clear ordering of cellulose nanocrystals on the surface and (d) the helicoidal assembly of CNC with a defined pitch, $p$, within the particle.

smaller microdroplets (50 $\mu \mathrm{m}$ in diameter) are employed to minimize the effects of buckling on the particle structure. ${ }^{35}$ Similarly, to the case of planar CNC films, ${ }^{20}$ the chiral nematic nature can be maintained, evidenced by the clear helicoidal structure observed in the scanning electron microscopy (SEM) image reported in Figure 4d. Moreover, the value of the pitch measured directly from such images, closely matches the extrapolated confined pitch for a CNC concentration near $100 \%$, as indicated by the dashed blue line in Figure 3. In contrast, casting a planar film via slow evaporation using the same suspension (Figure S11, Supporting Information) results in a cholesteric pitch consistent with the standard power law behavior (dashed red line).

\section{CONCLUSIONS}

In conclusion, we have fabricated bioinspired hierarchical cellulose structures over multiple length scales by controlling the self-assembly process of cellulose nanocrystals in micronsized droplets. The obtained droplets are sufficiently monodisperse to allow for local study of the self-assembly of the CNC water suspension. It is further demonstrated that this 
geometrical confinement leads to a drastic effect on the selfassembly of cellulose nanocrystals. This allows for the construction of an "evolution pitch diagram" that provides a tool to investigate the suspension behavior for a large range of concentrations. To our knowledge, this has not been reported so far, due to the fact that kinetic arrest usually leads to unreliable pitch values for high concentration. ${ }^{30}$

\section{EXPERIMENTAL METHODS}

Materials. Hexadecane (99\%) and Span 80 were purchase from Sigma-Aldrich and Fluka, respectively, and were used without further purification. The initial suspension of $14.5 \mathrm{wt} \%$ cellulose nanocrystals was prepared from filter paper, as described below, with subsequent formulations diluted with deionized water (Millipore Milli-Q gradient A10, resistivity $>18 \mathrm{M} \Omega \cdot \mathrm{cm}^{-1}$ ).

Instrumentation. Microdroplets were imaged in transmission using a Vision Research Phantom Miro ex4-M fast camera, attached to an Olympus IX-71 inverted microscope (10-64X objectives). Polarized optical microscopy was performed in transmission with crossed polarizers. In order to indicate $\mathrm{CNC}$ orientation, a sensitive tint plate (Olympus U-TP530) was additionally inserted between the crossed polarizers. SEM images were acquired using a Zeiss Leo Gemini $1530 \mathrm{VP}$ system, working at $90^{\circ}$ with respect to the electron beam. SEM samples were mounted on aluminum stubs using conductive carbon tape and, to minimize surface charging, sputtered with a 5-10 nm layer of $\mathrm{Au} / \mathrm{Pd}$ (Emitech K550; $I=55 \mathrm{~mA}$ for $10 \mathrm{~s}$ ). The acceleration voltage used was $2.0 \mathrm{kV}$, and the working distance was $1-2 \mathrm{~mm}$. Atomic force microscopy (AFM) images were acquired with an Agilent 5500, collected in tapping mode (OTESPA-R3 tip) and at room temperature over a $25 \mu \mathrm{m}^{2}$ area. AFM samples were prepared by drop-casting $10 \mu \mathrm{L}$ of a diluted CNC suspension on to poly(L-lysine)-functionalized mica. After deposition, the samples were rinsed with deionized water and dried under nitrogen flow.

Cellulose Nanocrystal Suspension. Cellulose nanocrystals were obtained from the hydrolysis of Whatman No. 1 cellulose filter paper $(30 \mathrm{~g})$ with sulfuric acid $(64 \mathrm{wt} \%, 420 \mathrm{~mL})$ at $64{ }^{\circ} \mathrm{C}$ for $30 \mathrm{~min}$, before being quenched using Milli- $\mathrm{Q}$ ice and water. Soluble cellulose residues and acid were removed by centrifugation (three steps at $20000 \mathrm{~g}$ for $20 \mathrm{~min}$ ) and dialysis against deionized water (MWCO 12$14 \mathrm{kDa}$ membrane), and a stable suspension of $[\mathrm{CNC}]=2.2 \mathrm{wt} \%$ was obtained. Conductivity titration against sodium hydroxide indicated $\left[\mathrm{SO}_{4}{ }^{2-}\right]=205 \mathrm{mmol} \cdot \mathrm{kg}^{-1}$ of $\mathrm{CNC}^{36}$ The suspension was tipsonicated in an ice bath (Fisherbrand Ultrasonic disintegrator $500 \mathrm{~W}$, amplitude $30 \% \max 8200 \mathrm{~J} \cdot \mathrm{g}^{-1}$ of CNC) and vacuum-filtered $(8.0 \mu \mathrm{m}$ then $0.8 \mu \mathrm{m}$ nitrocellulose, Sigma-Aldrich).

The suspension was concentrated by heating at $60{ }^{\circ} \mathrm{C}$ in a water bath for $12 \mathrm{~h}$, resulting in a $14.5 \mathrm{wt} \%(\sim 9.4 \% \mathrm{v} / \mathrm{v})$ suspension of CNC. The ionic content of this concentrated suspension was deduced from conductometric titration against sodium hydroxide, complemented by $\mathrm{pH}$ measurements before and after potassium chloride addition, and corresponds to the following: $36 \%$ surface charge loss by CNC partial desulfation $\left(\left[\mathrm{SO}_{4}{ }^{2-}\right]=131 \mathrm{mmol} \cdot \mathrm{kg}^{-1}\right.$ of $\left.\mathrm{CNC}\right)\left[\mathrm{CNC}^{-}\right]$ $=19 \mathrm{mM}$, the release of $\left[\mathrm{SO}_{4}^{2-}\right]_{\text {free }}=10.7 \mathrm{mM},\left[\mathrm{H}^{+}\right]_{\text {free }}=18.7 \mathrm{mM}$ and $\left[\mathrm{H}^{+}\right]_{\text {total }}=40.4 \mathrm{mM}$. Planar films of $\mathrm{CNC}$ were prepared by casting $1.0 \mathrm{~mL}$ of a $2.0 \mathrm{wt} \% \mathrm{CNC}$ suspension in a $3.5 \mathrm{~cm}$ diameter polystyrene petri dish, before being allowed to evaporate under ambient conditions.

Phase Diagram and Pitch Measurement in Capillaries. The initial $14.5 \mathrm{wt} \% \mathrm{CNC}$ suspension was diluted with deionized water using a high precision scale, vortexed, and transferred to a flat capillary of sufficiently large inner dimensions to eliminate any confinement effects $(1.00 \times 10.00 \times 50 \mathrm{~mm})$ and sealed with glass plates and nail polish. The self-assembly was observed after 4 days and later after 95 days with no noticeable change of the critical concentrations (Figure S12). The cholesteric pitch in the glass capillaries was then determined using (i) polarized optical microscopy (TPlan Nikon, 20× $(\mathrm{NA}=0.30$, $\mathrm{WD}=30 \mathrm{~mm})$ and $50 \times(\mathrm{NA}=0.40, \mathrm{WD}=22 \mathrm{~mm})$ objectives, Figure S13, Supporting Information); the pitch was measured as twice the period of the fingerprint pattern, taking either an average over 10 pitch distances or using a fast Fourier transform of the image processed with ImageJ; (ii) laser diffraction performed using a laser $(\lambda=531.8 \mathrm{~nm})$ and observing the diffraction pattern in transmission (as exemplified in Figures S14 and S15, Supporting Information). The pitch was derived using Bragg's law, as adapted by Kahn to include Snell law correction. ${ }^{37}$ In order to account for possible sample inhomogeneity in the vertical dimension, the pitch was measured at regular intervals throughout the anisotropic phase and is reported in Figure S16 in the Supporting Information.

Droplet-Based Microfluidics. Monodisperse water-in-oil microdroplets were generated within a hydrophobic flow-focusing microfluidic device. These were manufactured from PDMS via soft lithography, whereby the microchannel network was (i) designed in silico (AutoCAD), (ii) printed as a negative photomask, and (iii) transferred onto a silicon wafer spin-coated with SU-8 photoresist via UV-photolithography to form a mold. PDMS and the cross-linker (Sylgard 184 elastomer kit, Dow Corning) in a 10:1 ratio were poured onto this mold and allowed to stand overnight at $70{ }^{\circ} \mathrm{C}$. The PDMS layer, imprinted with the microfluidic channel design, was removed, and using a biopsy punch $(1.0 \mathrm{~mm})$, inlets and an outlet were formed. The imprinted PDMS and a glass substrate were exposed to oxygen plasma for $8 \mathrm{~s}$ and then pressed together to seal the microfluidic channels.

To study the self-assembly of the CNC suspension within the microdroplet, a $200 \mu \mathrm{m}$ wide flow-focusing junction (A) was employed with a channel depth of $80 \mu \mathrm{m}$. For structural analysis of solid microparticles a smaller flow-focusing junction $(43 \times 43 \mu \mathrm{m}, \mathrm{B})$ was employed to generate the templating microdroplets. To render the channels fluorophilic, they were immediately flushed with a $0.5 \% \mathrm{v} / \mathrm{v}$ solution of trichloro $(1 \mathrm{H}, 1 \mathrm{H}, 2 \mathrm{H}, 2 \mathrm{H}$-perfluorooctyl)silane in Fluorinert FC-40 (3M) and subsequently cured at $120{ }^{\circ} \mathrm{C}$ overnight. This hydrophobic surface modification was also applied to glass slides that were used in "shrinking droplet" studies, ensuring low wetting of the surface by the aqueous microdroplets.

To generate microdroplets, the continuous oil phase and the discrete aqueous phase were injected into the microfluidic device via two syringe pumps (PHD 2000, Harvard Apparatus) with controlled flow rates of 200 and $80 \mu \mathrm{L} \cdot \mathrm{h}^{-1}$, respectively. At the intersection, the shear forces caused the formation of aqueous droplets in oil (typically for a $7.3 \mathrm{wt} \% \mathrm{CNC}$ suspension: $\varnothing_{\mathrm{A}}=139.5 \pm 2.5 \mu \mathrm{m}$ and $\varnothing_{\mathrm{B}}=50.1$ $\pm 0.6 \mu \mathrm{m}$; Figure S17, Supporting Information). The continuous phase comprised the organic oil, hexadecane, with 2.0 wt \% Span 80 surfactant. The dispersed phase consisted of an aqueous suspension of cellulose nanocrystals; this was diluted as appropriate with deionized water from an initial $14.5 \mathrm{wt} \% \mathrm{CNC}$ suspension and vortexed to homogenize the sample prior to injection into the microfluidic device. Once the microemulsion was generated, it exited the microfluidic device through microbore polythene tubing $\left(\varnothing_{\mathrm{I}}=380 \mu \mathrm{m}, l \approx 10 \mathrm{~cm}\right)$ and was collected onto a microscope slide.

The aqueous phase was allowed to slowly diffuse into the oil at RTP until solid microparticles were formed. During droplet shrinkage, a linear decrease in the droplet diameter was observed (Figure S18, Supporting Information). This was typically in the range of 10-20 $\mu \mathrm{m} \cdot \mathrm{h}^{-1}$ for the large droplets $\left(\varnothing_{\mathrm{A}}\right)$ and increased to $25 \mu \mathrm{m} \cdot \mathrm{h}^{-1}$ for the smaller droplets $\left(\varnothing_{\mathrm{B}}\right)$ reported in Figure 4 (see Supporting Information).

Microparticle Analysis. Residual surfactant was removed from the dry microparticles by washing with $n$-hexane prior to imaging by SEM. To image the interior of a droplet, it was fractured using the following protocol: the particles (on a substrate) were first placed in a nitrogen atmosphere, cooled in liquid nitrogen, and finally mechanically crushed. The low temperature made the droplets more brittle, while the low humidity inhibited condensation of water.

\section{ASSOCIATED CONTENT}

\section{Supporting Information}

The Supporting Information is available free of charge on the ACS Publications website at DOI: 10.1021/acsnano.6b03355. 
AFM of CNC rods, additional images of cholesteric droplets, evolution pitch diagrams, comparative SEM of cross sections of films and microdroplets prepared from the same suspension, comparison of pitch measurements by laser diffraction and optical microscopy, droplet size distribution and their drying rate; with supplementary discussions on the evaporation process, phase diagram, pitch diagram, and modeling of the optical behavior of cholesteric droplets (PDF)

Video S1 (AVI)

Video S2 (AVI)

\section{AUTHOR INFORMATION}

\section{Corresponding Author}

*E-mail:sv319@cam.ac.uk.

\section{Author Contributions}

${ }^{\dagger}$ R.M.P. and B.F.-P. contributed equally to this work.

\section{Notes}

The authors declare no competing financial interest.

Additional data related to this publication is available at the University of Cambridge data repository (http://dx.doi.org/10. 17863/CAM.1233).

\section{ACKNOWLEDGMENTS}

This work was supported by a BBSRC David Phillips fellowship [BB/K014617/1], the Isaac Newton Trust Cambridge [76933], and the European Research Council [ERC-2014-STG H2020 639088].

\section{REFERENCES}

(1) Wegst, U. G. K.; Bai, H.; Saiz, E.; Tomsia, A. P.; Ritchie, R. O. Bioinspired Structural Materials. Nat. Mater. 2015, 14, 23-36.

(2) Aizenberg, J.; Fratzl, P. Biological and Biomimetic Materials. Adv. Mater. 2009, 21, 387-388.

(3) Ortiz, C.; Boyce, M. C. Bioinspired Structural Materials. Science 2008, 319, 1053-1054.

(4) Neville, A. C. Biology of Fibrous Composites; Cambridge University Press, 1993.

(5) Aguilar Gutierrez, O. F.; Rey, A. D. Chiral Graded Structures in Biological Plywoods and in the Beetle Cuticle. Colloids Interface Sci. Commun. 2014, 3, 18-22.

(6) Sharma, V.; Crne, M.; Park, J. O.; Srinivasarao, M. Structural Origin of Circularly Polarized Iridescence in Jeweled Beetles. Science 2009, 325, 449-451.

(7) Wilts, B. D.; Whitney, H. M.; Glover, B. J.; Steiner, U.; Vignolini, S. Natural Helicoidal Structures: Morphology, Self-Assembly and Optical Properties. Mater. Today: Proceedings 2014, 1, 177-185.

(8) Vignolini, S.; Moyroud, E.; Glover, B. J.; Steiner, U. Analysing Photonic Structures in Plants. J. R. Soc., Interface 2013, 10, 20130394.

(9) Reis, D.; Vian, B. Helicoidal Pattern in Secondary Cell Walls and Possible Role of Xylans in Their Construction. C. R. Biol. 2004, 327, 785-790.

(10) Glotzer, S. C. Assembly Engineering: Materials Design for the 21 st Century (2013 P.v. Danckwerts Lecture). Chem. Eng. Sci. 2015, 121, 3-9.

(11) Pokroy, B.; Kang, S. H.; Mahadevan, L.; Aizenberg, J. SelfOrganization of a Mesoscale Bristle into Ordered, Hierarchical Helical Assemblies. Science 2009, 323, 237-240.

(12) Dumanli, A. G.; van der Kooij, H. M.; Kamita, G.; Reisner, E.; Baumberg, J. J.; Steiner, U.; Vignolini, S. Digital Color in Cellulose Nanocrystal Films. ACS Appl. Mater. Interfaces 2014, 6, 12302-12306. (13) Sanchez, C.; Arribart, H.; Guille, M. M. G. Biomimetism and Bioinspiration as Tools for the Design of Innovative Materials and Systems. Nat. Mater. 2005, 4, 277-288.
(14) Vignolini, S.; Rudall, P. J.; Rowland, A. V.; Reed, A.; Moyroud, E.; Faden, R. B.; Baumberg, J. J.; Glover, B. J.; Steiner, U. Pointillist Structural Color in Pollia Fruit. Proc. Natl. Acad. Sci. U. S. A. 2012, 109, 15712-15715.

(15) Theberge, A. B.; Courtois, F.; Schaerli, Y.; Fischlechner, M.; Abell, C.; Hollfelder, F.; Huck, W. T. S. Microdroplets in Microfluidics: An Evolving Platform for Discoveries in Chemistry and Biology. Angew. Chem., Int. Ed. 2010, 49, 5846-5868.

(16) Humar, M.; Muševič, I. 3D Microlasers from Self-Assembled Cholesteric Liquid-Crystal Microdroplets. Opt. Express 2010, 18, 26995.

(17) Kim, J.-H.; Shim, B. S.; Kim, H. S.; Lee, Y.-J.; Min, S.-K.; Jang, D.; Abas, Z.; Kim, J. Review of Nanocellulose for Sustainable Future Materials. International Journal of Precision Engineering and Manufacturing-Green Technology 2015, 2, 197-213.

(18) Eichhorn, S. J.; Dufresne, A.; Aranguren, M.; Marcovich, N. E.; Capadona, J. R.; Rowan, S. J.; Weder, C.; Thielemans, W.; Roman, M.; Renneckar, S.; Gindl, W.; Veigel, S.; Keckes, J.; Yano, H.; Abe, K.; Nogi, M.; Nakagaito, A. N.; Mangalam, A.; Simonsen, J.; Benight, A. S.; Bismarck, A.; Berglund, L. A.; Peijs, T. Review: Current International Research into Cellulose Nanofibres and Nanocomposites. J. Mater. Sci. 2010, 45, 1-33.

(19) Revol, J. F.; Bradford, H.; Giasson, J.; Marchessault, R. H.; Gray, D. G. Helicoidal Self-Ordering of Cellulose Microfibrils in Aqueous Suspension. Int. J. Biol. Macromol. 1992, 14, 170-172.

(20) Revol, J. F.; Godbout, L.; Gray, D. G. Solid Self-Assembled Films of Cellulose with Chiral Nematic Order and Optically Variable Properties. J. Pulp Pap. Sci. 1998, 24, 146-149.

(21) Park, J. H.; Noh, J.; Schütz, C.; Salazar-Alvarez, G.; Scalia, G.; Bergström, L.; Lagerwall, J. P. F. Macroscopic Control of Helix Orientation in Films Dried from Cholesteric Liquid-Crystalline Cellulose Nanocrystal Suspensions. ChemPhysChem 2014, 15, 14771484.

(22) Roman, M.; Gray, D. G. Parabolic Focal Conics in SelfAssembled Solid Films of Cellulose Nanocrystals. Langmuir 2005, 21, 5555-5561.

(23) Beck, S.; Bouchard, J.; Berry, R. Controlling the Reflection Wavelength of Iridescent Solid Films of Nanocrystalline Cellulose. Biomacromolecules 2011, 12, 167-172.

(24) Giese, M.; Blusch, L. K.; Khan, M. K.; Hamad, W. Y.; MacLachlan, M. J. Responsive Mesoporous Photonic Cellulose Films by Supramolecular Cotemplating. Angew. Chem., Int. Ed. 2014, 53, $8880-8884$.

(25) Kelly, J. A.; Shukaliak, A. M.; Cheung, C. C. Y.; Shopsowitz, K. E.; Hamad, W. Y.; MacLachlan, M. J. Responsive Photonic Hydrogels Based on Nanocrystalline Cellulose. Angew. Chem., Int. Ed. 2013, 52, 8912-8916.

(26) Jativa, F.; Schütz, C.; Bergström, L.; Zhang, X.; Wicklein, B. Confined Self-Assembly of Cellulose Nanocrystals in a Shrinking Droplet. Soft Matter 2015, 11, 5374-5380.

(27) Noh, J.; Reguengo De Sousa, K.; Lagerwall, J. P. F. Influence of Interface Stabilisers and Surrounding Aqueous Phases on Nematic Liquid Crystal Shells. Soft Matter 2016, 12, 367-372.

(28) Noh, J.; Liang, H.-L.; Drevensek-Olenik, I.; Lagerwall, J. P. F. Tuneable Multicoloured Patterns from Photonic Cross-Communication Between Cholesteric Liquid Crystal Droplets. J. Mater. Chem. C 2014, 2, 806-810.

(29) Muševič, I. Nematic Colloids, Topology and Photonics. Philos. Trans. R. Soc., A 2013, 371, 20120266.

(30) Schütz, C.; Agthe, M.; Fall, A. B.; Gordeyeva, K.; Guccini, V.; Salajkova, M.; Plivelic, T. S.; Lagerwall, J. P. F.; Salazar-Alvarez, G.; Bergström, L. Rod Packing in Chiral Nematic Cellulose Nanocrystal Dispersions Studied by Small-Angle X-Ray Scattering and Laser Diffraction. Langmuir 2015, 31, 6507-6513.

(31) Beck, S.; Bouchard, J. Auto-Catalyzed Acidic Desulfation of Cellulose Nanocrystals. Nord. Pulp Pap. Res. J. 2014, 29, 006-014.

(32) Sen, D.; Mazumder, S.; Melo, J. S.; Khan, A.; Bhattyacharya, S.; D'Souza, S. F. Evaporation Driven Self-Assembly of a Colloidal 
Dispersion During Spray Drying: Volume Fraction Dependent Morphological Transition. Langmuir 2009, 25, 6690-6695.

(33) Straley, J. P. Theory of Piezoelectricity in Nematic Liquid Crystals, and of the Cholesteric Ordering. Phys. Rev. A: At., Mol., Opt. Phys. 1976, 14, 1835-1841.

(34) Honorato-Rios, C.; Kuhnhold, A.; Bruckner, J. R.; Dannert, R; Schilling, T.; Lagerwall, J. P. F. Equilibrium Liquid Crystal Phase Diagrams and Detection of Kinetic Arrest in Cellulose Nanocrystal Suspensions. Front. Mater. 2016, 3, 21.

(35) Cerda, E.; Mahadevan, L. Geometry and Physics of Wrinkling. Phys. Rev. Lett. 2003, 90, 074302.

(36) Beck, S.; Methot, M.; Bouchard, J. General Procedure for Determining Cellulose Nanocrystal Sulfate Half-Ester Content by Conductometric Titration. Cellulose 2015, 22, 101-116.

(37) Kahn, F. J. Electric-Field-Induced Color Changes and Pitch Dilation in Cholesteric Liquid Crystals. Phys. Rev. Lett. 1970, 24, 209212. 